\title{
Trauma Activation and Substance Use in an Urban Trauma Center
}

\author{
Derek Titus ${ }^{1,2}$, Areta Kowal-Vern',3, John Porter $^{4,5}$, Marc R. Matthews ${ }^{3,5}$, Philomene Spadafore5, \\ Sydney Vail ${ }^{5}$
}

${ }^{1}$ College of Osteopathic Medicine of the Pacific-Northwest, Lebanon, USA

${ }^{2}$ Department of Research, Valleywise Health Medical Center, Phoenix, USA

${ }^{3}$ Arizona Burn Center, Valleywise Health Medical Center, Phoenix, USA

${ }^{4}$ Department of Physical Medicine \& Rehabilitation, Valleywise Health Medical Center, Phoenix, USA

${ }^{5}$ Department of Surgery, Valleywise Health Medical Center, Phoenix, USA

Email: titus085@umn.edu, akvern@hotmail.com, John_Porter@dmgaz.org,`Marc_Matthews@dmgaz.org,

Philomene.Spadafore@valleywisehealth.org,Sydney_Vail@dmgaz.org

How to cite this paper: Titus, D., Kowal-Vern, A., Porter, J., Matthews, M.R., Spadafore, P. and Vail, S. (2021) Trauma Activation and Substance Use in an Urban Trauma Center. Surgical Science, 12, 53-66. https://doi.org/10.4236/ss.2021.123008

Received: January 28, 2021

Accepted: March 7, 2021

Published: March 10, 2021

Copyright (c) 2021 by author(s) and Scientific Research Publishing Inc. This work is licensed under the Creative Commons Attribution International License (CC BY 4.0).

http://creativecommons.org/licenses/by/4.0/

\section{(c) (i) Open Access}

\begin{abstract}
Background: Alcohol and drug screens in trauma patients are endorsed by the American College of Surgeons Committee on Trauma. The objective was to determine the characteristics and frequency of alcohol and poly-drug combinations in patients triaged by Trauma Team Activation (TTA) codes. Methods: A retrospective study of a Level I hospital trauma registry was initiated with 14,972 patients of whom 7028 (47\%) were Substance Users (SU). There were 10,369 TTA patients: 5396 (52\%) were SU, and 4603 (31\%) non-TTA patients, of whom 1632 (35.5\%) were SU. Inclusion criteria were: age $\geq 14$ years old, blood alcohol level and Urine Drug Screen (UDS) documentation, Injury Severity Score (ISS), TTA code, hospital charges and Length of Hospitalization (LOS). Statistical analyses were performed with one-way ANOVA, Maximum Likelihood chi squared, and Mann-Whitney U tests. Results: The median ISS was 5 for both groups. TTA patients were younger than non-TTA patients (median 33 years versus 41 years). Mortality was significantly higher for TTA (4.7\%) than for non-TTA (0.7\%) patients. From 2010-2013 to 20142018 , all substance users had a significant decrease in alcohol only use $58.0 \%$ to $42.0 \%)$ with an increase in single drug $(42.0 \%$ to $58.0 \%)$ and poly-drug use (36.9\% to $63.1 \%), p<0.0001$. For all SU patients (TTA and non-TTA), the major single drugs used were opiates (19.9\%), cannabinoids (THC) (15.2\%), benzodiazepines (7.7\%), amphetamines (6.6\%), and cocaine (2.5\%). The major poly-drugs were: opiates/THC (4.7\%), opiates/benzodiazepines (4.6\%), THC/amphetamines (3.1\%), THC/benzodiazepines (2.2\%). Conclusions: By trauma activation code designation, the TTA group had most of the positive alcohol and drug screens in this study. Since the change in mandated opioid
\end{abstract}


prescription practices in 2014, this study showed increased poly-drug use (two to six-drug combinations) in urban trauma patients between 2014-2018. Opioids and cannabinoids continued to be the most frequently used drugs singly and in combinations.

\section{Keywords}

Drugs, Alcohol, Trauma

\section{Introduction}

Alcohol, prescription and illicit drugs such as opiates, cannabinoids (THC, marijuana), and cocaine have been implicated in trauma injuries for decades. In the United States, many cases from accredited trauma and burn centers are registered in the National Trauma Data Bank (NTDB) with no recorded positive or negative alcohol or urine drug screens [1] [2] [3] [4] [5]. As a result, substance use research studies based on NTDB data or trauma registries that provide the data for the NTDB may have an inherent selection and compliance bias on this issue [1] [2] [3] [4] [5]. Table 1 shows a comparison of alcohol and drug screen demographic results in the NTDB, the literature and the present study [4] [5]. All substance users cannot be identified by sight only; trauma protocol non-compliance

Table 1. Comparison of NTDB, literature, and current study substance use screens.

\begin{tabular}{|c|c|c|c|c|c|}
\hline & $\begin{array}{c}\text { NTDB } \\
2010[6]\end{array}$ & $\begin{array}{c}\text { NTDB } \\
2016 \text { [7] }\end{array}$ & $\begin{array}{c}\text { Langdorf } \\
2002[5]\end{array}$ & $\begin{array}{l}\text { Dunham } \\
2012[4]\end{array}$ & $\begin{array}{c}\text { This study } \\
2021\end{array}$ \\
\hline Alcohol \# (\%) cases & 681,990 & 861,888 & 170 & 572 & 14,972 \\
\hline \# Tested & $252,781(38)$ & $337,678(39)$ & $144(85)$ & $338(59)$ & $10,791(72)$ \\
\hline \# Positive & $98,517(14.5)$ & $114,296(13.3)$ & $48(28.2)$ & $89(15.6)$ & $3309(22.1)$ \\
\hline \# Negative & $154,264(22.6)$ & $233,482(27.1)$ & -- & $323(56.4)$ & $7482(50.0)$ \\
\hline \# Not tested & $296,761(43.5)$ & $514,110(59.6)$ & -- & $234(40.9)$ & *4179 (28) \\
\hline$\%$ Minimum Rate & 14.4 & 13.3 & 28.2 & 23.1 & 22.1 \\
\hline$\%$ Tested Rate & 39 & 33.8 & 33.3 & 26.4 & 30.7 \\
\hline$U D S \#(\%)$ cases & 681990 & 861888 & 170 & 572 & 14972 \\
\hline \# Tested & $155,039(22.3)$ & $194,555(23)$ & $144(85)$ & $232(41)$ & $6181(40)$ \\
\hline \# Positive & $79,149(11.6)$ & $10,584(12.0)$ & $40(23.5)$ & $67(11.7)$ & $5320(36)$ \\
\hline \# Negative & $75,890(11.1)$ & $90,571(10.5)$ & -- & $165(28.8)$ & $861(4.0)$ \\
\hline \# Not tested & $526,951(77.0)$ & $667,733(77.5)$ & -- & $340(59.4)$ & *8791 (55) \\
\hline$\%$ Minimum Rate & 11.6 & 12.0 & 23.5 & 11.7 & 35.5 \\
\hline$\%$ Tested Rate & 51.1 & 53.2 & 27.8 & 28.9 & -- \\
\hline
\end{tabular}

Table modified from Dunham ${ }^{4}$ \# = number; NTDB = National Trauma Data Bank; UDS = urine drug screen; ${ }^{*}$ consisted of negative alcohol and drug screens, and screen variables with no documentation; Minimum rate is the number of positive tests divided by the total patients (tested and not tested); Tested rate is the number of positive tests divided by the number of patients tested. 
especially for the Urine Drug Screen (UDS) may have become discretionary, based on cost, insurance, legal, privacy issues, and logistics [1]-[5] [8]. The Trauma Team Activation (TTA) code that is used to assess patient severity of injury is a marker inclusive of all patients for required medical-financial triage [9].

The TTA code dictates charges for medical care categories and personnel required by trauma patients upon arrival in a medical facility. In 1987, the American College of Surgeons guidance for trauma centers was three "domains" of criteria for field facility triage with provisions for comorbidity and age extremes: physiologic, anatomic, and injury mechanism [9]. From their research, Dunham and Langdorf recommended that all TTA patients should be tested for alcohol and drug screens on arrival, and non-TTA patients on a discretionary basis, or when specifically ordered by the physician [4] [5]. With the current opioid crisis, overdose deaths, and Covid-19 pandemic, substance use risk is accelerated due to increased stress, medical and psychological co-morbidities, with social and economic challenges. Increased alcohol and drug screens will become imperative for treatment and prevention not only in trauma but all medical specialties [10] [11]. Trauma Team Activation (TTA) protocols that include alcohol and substance screens in coded patients may decrease the selection and compliance bias present in trauma registries. The objective of this study was to determine the demographic characteristics and drug combinations (alcohol/drugs/poly-drug use) of adult TTA and non-TTA patients to determine if mainly the injured substance users were tagged for the RED, Yellow, and Green TTA codes. Our hypothesis was that most patients with substance use would be coded for trauma team activation.

\section{Methods}

\subsection{Sample Population}

This was a retrospective trauma registry analysis of illicit and prescribed substance usage at an urban adult and pediatric Level 1 trauma center (January 1, 2010 to December 31, 2018). Of the 19,547 original cases, the exclusion criteria were: children < 14-years-old (3096, 16.0\%), and patients with missing data (4575, 23.4\%). Of the 14972 remaining cases, there were 10,369 (69.3\%) TTA patients, of whom 5396 (52\%) were substance users (SU); of the 4603 (30.6\%) non-TTA patients, 1632 (35.5\%) were SU. The inclusion criteria were: age $\geq 14$-years, Injury Severity Score (ISS), length of stay (LOS), hospital charges, recorded insurance providers, gender, injury type (blunt vs penetrating), survival, ethnicity, and either a positive recorded alcohol blood level or urine drug screen (UDS) or both. This study was approved on May 11, 2018 by the Institutional Review Board of Valleywise Health Medical Center (former Maricopa Integrated Health System).

\subsection{Urine Drug Screen}

Qualitative results for urinary drugs of use were obtained by Vitros 5600 and 
Vitros 4600 testing systems (Ortho Clinical Diagnostics [OCD], Raritan, NJ). Only FDA-approved reagents manufactured by Immunalysis, (Thermo Fisher, Waltham, MA) and OCD were used to complete a screen of up to 15 compounds in a single panel. Drugs tested were: amphetamine, barbiturates, benzodiazepines (BZD), cocaine, tetrahydrocannabinols (THC), methadone, methamphetamine, opiate (OPI 300), oxycodone, and phencyclidine. Drugs such as tramadol, 6-AM, buprenorphine, 6-Acetyl M, methylenedioxymethamphetamine, and fentanyl were listed in the trauma registry "OTHER" drug category. Prescription or illicit drug status was not determined for opiate, BZD, THC or amphetamine use.

\subsection{Blood Alcohol Testing}

Blood serum alcohol levels were determined on the Ortho Clinical Diagnostics (OCD) Vitros 5600 (Ortho Diagnostics, Raritan, NJ) and utilized the OCD reagent with MicroSlide Technology (Ortho Diagnostics, Raritan, NJ). This was not a study on the legal limits of alcohol presence or intoxication. All blood alcohol levels, BAC $\geq 0.01 \mathrm{~g} / \mathrm{dl}$, were included in the study groups since any level rendered the screen as positive.

\subsection{Statistical Analysis}

Statistical analyses used SAS ${ }^{\circledR}$ software (SAS Institute, Cary NC) for descriptive statistics, comparison of TTA versus non-TTA patients, and substance use cohorts: alcohol only (alcohol); alcohol + drugs (alcohol + drugs); single drug only (single drug); poly-drugs only (poly-drugs). Miscellaneous cases were those with negative alcohol and drug screens, and screen variables with no documentation; they were in both the TTA and non-TTA coded groups but separate from the drug cohorts. One-way ANOVA with a Tukey post-hoc test determined the median, interquartile range (IQR) for age, ISS score, length of stay (LOS), and hospital charges. Maximum Likelihood chi-squared analysis was performed on ISS, ethnicity, gender, trauma etiology (blunt versus penetrating), age subgroups $(<20,20-54,55+$ years), payers, mortality. Because the Opioid Task Force was organized in 2014 to combat the national overdose epidemic, substance usage time frames were compared as 2010-2013 versus 2014-2018. Mann-Whitney U test compared the drug groups and time frames for age, LOS, ISS, and hospital charges. Odds ratios were determined for TTA versus non-TTA patients based on ISS subsets $(<9 ; \geq 9)$, hospital charges (United States dollars [\$USD] $\geq 50,000$ ), etiology (blunt versus penetrating), hospital stay ( $<$ day; $\geq$ a day), suicide, mortality, and insurance providers using a Wald chi-squared test for significance. In addition, odds ratios were also performed for the non-TTA central nervous system (CNS) component versus the non-CNS cases). For each variable, a $p$ value $<$ 0.05 was considered significant.

\section{Results}

Most trauma injury (49.4\%) was sustained through motor vehicle collisions with 
other vehicles, objects, and pedestrians; less frequent were falls (12.3\%), pierced instruments (11.2\%), firearms (6.4\%) and other methods (20.7\%). Figure 1 shows the individual drug use annually in all patients. Regardless of whether the patients tested positive for substance use, the most severely acute patients, (2971, $19.8 \%$ ), were activated as life-threatening with TTA RED code; the YELLOW code activated 3184 (21.3\%) patients with non-life-threatening injuries. Level III GREEN was assigned to 4215 (28.2\%) patients, who did not belong in the RED or YELLOW codes but were candidates for either a trauma consultation or possible admission.

\subsection{TTA versus Non-TTA Patients}

Table 2 shows the demographic characteristics of TTA and non-TTA patients. Of the 14,972 patients, 7028 (47\%) were substance users (SU). There were 10,369 TTA patients, of whom 5396 (52\%) were SU, and 4603 (31\%) non-TTA patients, of whom 1632 (35.5\%) were SU. The TTA patients were younger, had higher numbers of penetrating and blunt trauma, and a higher mortality $(4.7 \%$ versus $0.7 \%)$ than the non-TTA population, $p<0.0001$. By ethnicity, TTA patients

Table 2. Demographic characteristics by trauma team activation codes.

\begin{tabular}{cccc}
\hline & TTA & Non-TTA & Total \\
\hline Number (\%) & $10,369(69.3)$ & $4603(30.7)$ & 14,972 \\
All Ages median (IQR) & $33.3(24.1,48.3)$ & $41.2(25.1,56.1)$ & $35.5(25.0,51.1)$ \\
<20 years \# (\%) & $1352(9.0)$ & $450(3.0)$ & $1802(12.0)$ \\
20 - 54.99 years \# (\%) & $7389(50.0)$ & $2904(19.0)$ & $10,293(69.0)$ \\
55+ years \# (\%) & $1682(11.0)$ & $1249(8.0)$ & $2877(19.0)$ \\
LOS (days) median (IQR) & $1.1(0.2,4.1)$ & $2.0(1.1,3.9)$ & $1.6(0.3,4.0)$ \\
M/F (number) & $6901 / 3468$ & $3387 / 1216$ & $10,288 / 4684$ \\
M/F Ratio & $2: 1$ & $3: 1$ & $2: 1$ \\
ISS score median (IQR) & $5.0(1.0,11.0)$ & $5.0(2.0,9.0)$ & $5.0(1.0,10.0)$ \\
Charges/patient (\$USD) & 42,377 & 38,732 & 41,517 \\
Median (IQR) & $(25,921,81,878)$ & $(22,012,70,110)$ & $(24,807,76,790)$ \\
Suicide \# (\%) & $315(2.1)$ & $123(0.8)$ & $438(2.9)$ \\
Mortality \# (\%) & $490(4.7)$ & $32(0.7)$ & $522(3.49)$ \\
Penetrating trauma \# (\%) & $1729(11.6)$ & $646(4.3)$ & $2375(16)$ \\
Blunt trauma \# (\%) & $8640(57.7)$ & $3957(26.4)$ & $12,597(84.0)$ \\
ISS $\geq 9$ \# (\%) & $3702(25.0)$ & $1291(8.6)$ & $4993(33.0)$ \\
ISS < 9 \# (\%) & $6667(44.5)$ & $3312(22.0)$ & $9979(67.0)$ \\
2010-2013 \# (\%) & $5252(35.1)$ & $1747(11.7)$ & $6999(46.8)$ \\
2014-2018 \# (\%) & $5117(34.2)$ & $2856(19.1)$ & $7973(53.3)$ \\
\hline
\end{tabular}

\# $(\%)=$ number (percent); median (interquartile range $\left(\mathrm{IQR}, 25^{\text {th }}\right.$ percentile, $75^{\text {th }}$ percentile); $\mathrm{TTA}=$ trauma team activation coded patients; non-TTA = non-TTA coded patients; ISS = injury severity score; LOS = length of stay; $\$ \mathrm{USD}=$ United States dollars; $\mathrm{M}=$ male; $\mathrm{F}=$ female. 


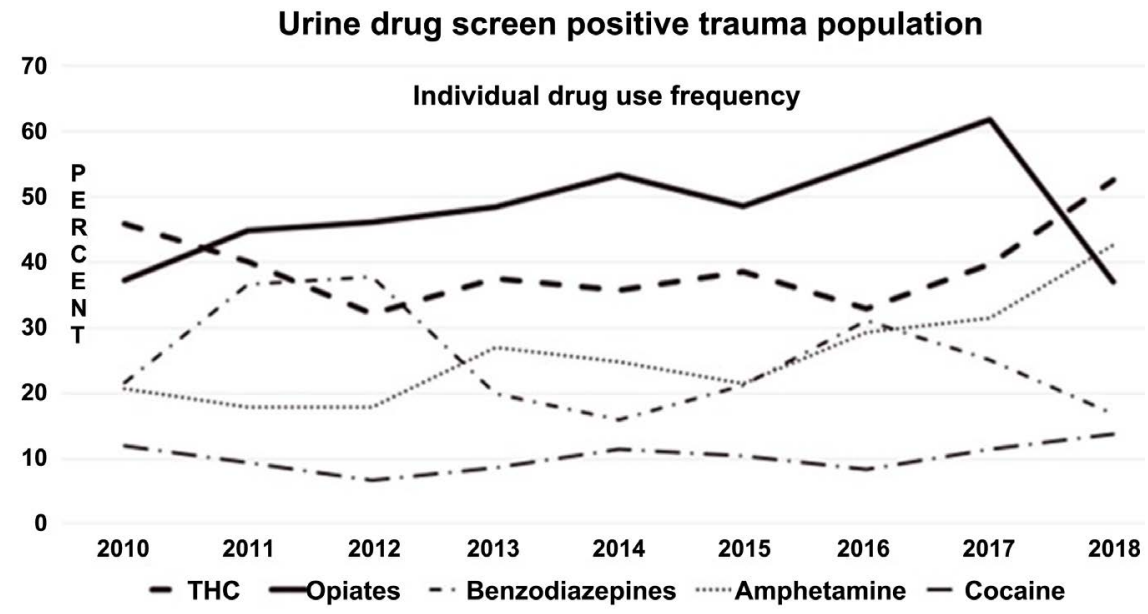

Figure 1. Annual individual drug use in all patients.

were like the non-TTA patients: 41.7\% Caucasian; 36.0\% Hispanic/Latino; 9.8\% American Indian and Alaskan natives; 9.5\% African American; 3.0\% Other. The most frequent payer was Medicaid 30.5\% for both groups. Other providers differed: private and commercial insurance covered $28.2 \%$ for the TTA versus $16.3 \%$ for the non-TTA patients; Medicare $7.4 \%$ versus $12.3 \%$; self-pay $20.0 \%$ versus $15.6 \%$.

Of the 4603 non-TTA patients, 3081 (67\%) had central nervous system (CNS) trauma; other patients had general, medical, and neurological conditions. The median ISS for this CNS subset was 5, the median (IQR) for LOS was 2.1 (1.2, $4.1)$ and age, $41(28.5,55.2)$. By cohort, $7.24 \%$ patients were with alcohol + drugs, $7.37 \%$ alcohol only, $12.2 \%$ single drug, $13.8 \%$ poly-drugs, and $59.5 \%$ with the miscellaneous cohort. They were more likely to be hospitalized for more than 24 hours (OR 3.58 [95\% CI 3.25 - 3.94], $p<0.0001$ ), had low mortality rates (OR 0.16 [95\% CI $0.11-0.25], p<0.0001$ ), and a higher likelihood of poly-drug use (OR 1.28 (95\% CI 1.14 - 1.44) than the non-CNS cases).

\subsection{Combined TTA and Non-TTA Patients with Positive Alcohol and Drug Screens}

Table 3 and Figure 2 depict the major demographic characteristics of the four categories for positive toxicology screened substance use: 1) alcohol only (alcohol) 1723 (24.5\%); 2) alcohol + drugs-1580 (22.5\%); 3) single drug-1981 (28.0\%); 4) poly-drugs-1744 (24.8\%). Patients in the alcohol + drugs group were significantly younger than those in the single drug and the alcohol groups, $p<0.0001$. LOS was significantly increased in the alcohol + drugs, single drug and poly-drug groups compared to the alcohol group, $p<0.0001$. The combined mortality for the SU group was $2.4 \%, p<0.001$. By ethnicity, the patients were similarly distributed within the cohorts: 2850 (40.6\%) Caucasian; 2199 (31.3\%) Hispanic and Latino; 1058 (15.1\%) Native American Indian and Alaskan; 763 (10.9\%) African American; 158 (2.3\%) Other. The ethnicity of the patients with positive alcohol and drug screens was like that seen in the TTA and non-TTA 
Table 3. Trauma patient demographic characteristics by drug cohorts.

\begin{tabular}{|c|c|c|c|c|c|}
\hline & Alcohol only & Alcohol + drugs & Single drug & Poly-drugs & ${ }^{*}$ Miscellaneous \\
\hline Number (\%) & 1723 & 1580 & 1981 & 1744 & 7944 \\
\hline All ages (years) median (IQR) & $36.1(27.0,50.0)$ & $32.5(25.1,45.0)$ & $35.0(25.0,51.0)$ & $33.6(25.1,46.7)$ & $37.0(24.0,54.0)$ \\
\hline$<20 \#(\%)$ & $91(0.6)$ & $113(0.8)$ & $232(1.6)$ & $162(1.1)$ & $1204(8.0)$ \\
\hline $20-54.99 \#(\%)$ & $1368(9.1)$ & $1317(8.8)$ & $1362(9.1)$ & $1395(9.32)$ & $4850(32.4)$ \\
\hline $55+\#(\%)$ & $263(15.3)$ & $150(1.0)$ & $387(2.6)$ & $187(1.3)$ & $1890(12.6)$ \\
\hline LOS (days) median (IQR) & $1.5(0.3,3.8)$ & $2.3(0.7,5.7)$ & $2.4(0.9,5.3)$ & $2.6(1.0,6.0)$ & $1.1(0.2,3.0)$ \\
\hline $\mathrm{M} / \mathrm{F}$ (number) & $1347 / 376$ & $1270 / 310$ & $1358 / 623$ & $1286 / 458$ & $5027 / 2917$ \\
\hline M/F Ratio & $3.6: 1$ & $4: 1$ & $2: 1$ & 2.8:1 & $1.7: 1$ \\
\hline ISS score median (IQR) & $5.0(1.0,10.0)$ & $5.0(2.0,12.0)$ & $5.0(2.0,12.0)$ & $5(2.0-11.0)$ & $4(1.0,9.0)$ \\
\hline Charges/patient (\$USD) & 41,905 & 48,338 & 52,229 & 56,220 & 36,066 \\
\hline Median (IQR) & $(26,673,74,030)$ & $(30,299,94,461)$ & $(33,680,101,973)$ & $(32,150,110,551)$ & $(21,806,61,890)$ \\
\hline Suicide \# (\%) & $54(0.4)$ & $60(0.4)$ & $74(0.5)$ & $54(0.4)$ & $196(1.3)$ \\
\hline Mortality \# (\%) & $62(0.41)$ & $33(0.22)$ & $51(0.34)$ & $20(0.13)$ & $356(2.4)$ \\
\hline Penetrating trauma \# (\%) & $331(2.2)$ & $408(2.7)$ & $247(1.7)$ & $355(2.4)$ & $1034(6.9)$ \\
\hline Blunt trauma \# (\%) & $1392(9.3)$ & $1172(7.8)$ & $1734(11.6)$ & $1389(9.3)$ & $6910(46.2)$ \\
\hline ISS $\geq 9 \#(\%)$ & $574(3.8)$ & $637(4.3)$ & $830(5.5)$ & $697(4.7)$ & $2255(15.1)$ \\
\hline ISS $<9 \#(\%)$ & $1149(7.7)$ & $9436.3)$ & $1151(7.7)$ & $1047(7.0)$ & $5689(38.0)$ \\
\hline TTA \# (\%) & $1390(9.3)$ & $1291(8.6)$ & $1516(10.1)$ & $1199(8.0)$ & $4973(33.2)$ \\
\hline Non-TTA \# (\%) & $333(2.2)$ & $289(1.9)$ & $465(3.1)$ & $545(3.6)$ & $2971(20.0)$ \\
\hline 2010-2013 \# (\%) & $999(6.7)$ & $840(5.6)$ & $832(5.6)$ & $643(4.3)$ & $3685(24.6)$ \\
\hline 2014-2018 \# (\%) & $724(4.9)$ & $740(4.8)$ & $1149(7.7)$ & $1101(7.4)$ & $4259(28.5)$ \\
\hline
\end{tabular}

$\#(\%)=$ number $\left(\right.$ percent); median (interquartile range $\left[\mathrm{IQR}, 25^{\text {th }}\right.$ percentile, $75^{\text {th }}$ percentile $]$ ); TTA $=$ trauma team activation coded patients; non-TTA $=$ not TTA coded patients; ${ }^{*}$ consisted of negative alcohol and drug screens, and screen variables with no documentation; ISS = injury severity score; LOS = length of stay; $\$$ USD = United States dollars; $M=$ male; $F=$ female.

\section{Distribution of patient alcohol and drug use by trauma team activation (TTA) and non-TTA code}

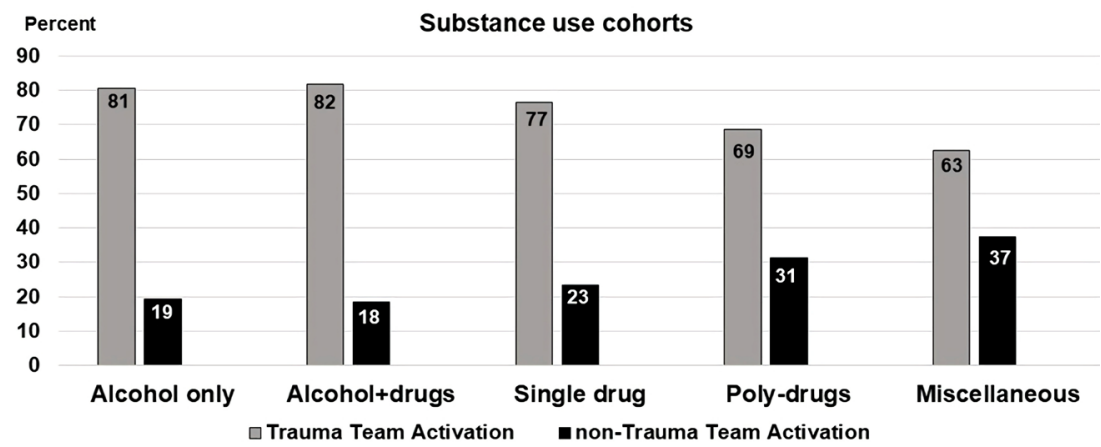

Figure 2. Four substance use cohorts and the miscellaneous group of negative alcohol and urine drug screens and undocumented screen variables.

patients. Median hospital charges/patient were in the United States dollars $(\$ \mathrm{USD}) \geq 50,000$ range. Major providers for all patients were: 2622 (37.3\%) Me- 
dicaid; 2426 (34.5\%) private/commercial/government/Workman's Comp; 1252 (17.8\%) Self-Pay; 493 (7.0\%) Medicare; 235 (3.4) Other. Figure 3 indicates that among the TTA coded patients, the highest number of RED codes was in the alcohol + drugs cohort, and the highest number of GREEN codes was in the "miscellaneous" cohort. There were statistical differences among the coded and cohort cases, $p<0.001$ (Pearson and Maximum Likelihood chi square tests). However, these were not reported due to the partial unknown status of the "miscellaneous" cohort in this study.

\subsection{Odds Ratio: TTA versus Non-TTA Patients}

The Odds Ratio indicated that there was a greater association of substance use (alcohol + drugs and alcohol alone), age $<55$ years, commercial insurance, and self-pay within the TTA group, than within the non-TTA group, Table 4. A significantly higher mortality was present in the TTA patients compared to the

Table 4. Odds ratios by TTA versus non-TTA patients.

\begin{tabular}{|c|c|c|c|}
\hline \multicolumn{4}{|c|}{ Odds Ratios by TTA versus Non-TTA } \\
\hline Variable & Odds Ratio & Wald CI & $P$ value \\
\hline Substance user (SU) & 1.98 & $(1.84,2.12)$ & $<0.0001$ \\
\hline Poly-drugs & 0.97 & $(0.87,1.08)$ & 0.6389 \\
\hline Single drug & 1.52 & $(1.36,1.70)$ & $<0.0001$ \\
\hline Alcohol + drugs & 2.12 & $(1.86,2.42)$ & $<0.0001$ \\
\hline Alcohol alone & 1.99 & $(1.75,2.25)$ & $<0.0001$ \\
\hline Age $<20$ years & 1.38 & $(1.24,1.55)$ & $<0.0001$ \\
\hline Age 20 to $<55$ years & 1.45 & $(1.35,1.56)$ & $<0.0001$ \\
\hline Age $\geq 55$ years & 0.50 & $(0.46,0.54)$ & $<0.0001$ \\
\hline LOS $<1$ day & 3.34 & $(3.08,3.62)$ & $<0.0001$ \\
\hline LOS $\geq 1$ day & 0.30 & $(0.28,0.32)$ & $<0.0001$ \\
\hline ISS rating $\geq 9$ & 1.42 & $(1.32,1.54)$ & $<0.0001$ \\
\hline Blunt trauma & 0.80 & $(0.73,0.89)$ & $<0.0001$ \\
\hline Penetrating trauma & 1.24 & $1.13,1.37$ & $<0.0001$ \\
\hline $\begin{array}{l}\text { Hospital charges } \\
\geq 50,000 \text { \$USD }\end{array}$ & 1.15 & $(1.07,1.24)$ & $<0.0001$ \\
\hline Suicide & 1.14 & $(0.92,1.41)$ & 0.2272 \\
\hline Mortality & 7.09 & $(4.95,10.15)$ & $<0.0001$ \\
\hline Medicaid & 0.98 & $(0.91,1.06)$ & 0.6719 \\
\hline Medicare & 0.57 & $(0.51,0.64)$ & $<0.0001$ \\
\hline Private/commercial & 1.91 & $(1.74,2.09)$ & $<0.0001$ \\
\hline Self-pay & 1.34 & $(1.23,1.48)$ & $<0.0001$ \\
\hline
\end{tabular}

LOS = length of stay; ISS = Injury severity score; TTA = trauma team activation code patients; non-TTA = non-trauma team activation code; $\$ \mathrm{USD}=$ United States dollars. 
Patient alcohol and drug use by trauma team activation (TTA) code

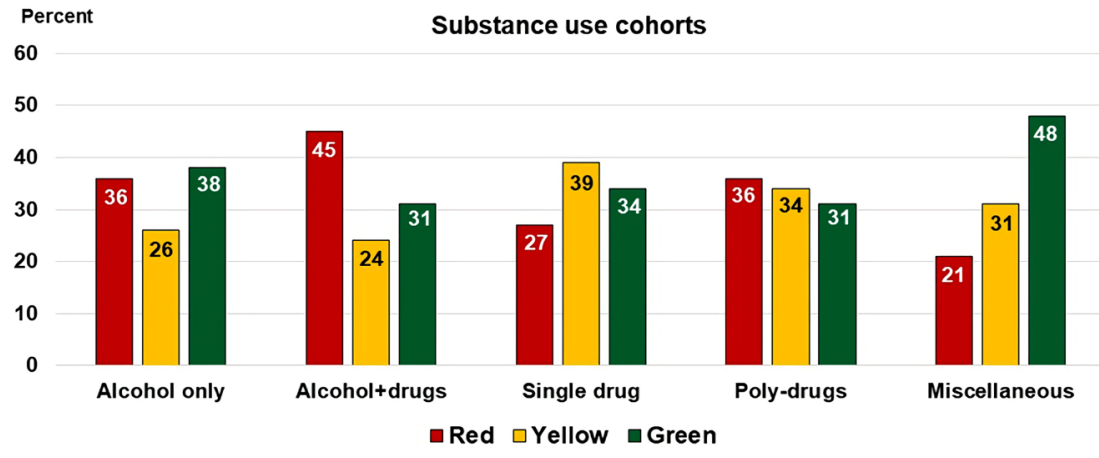

Figure 3. Trauma activation color-coded triage of the four substance use cohorts and the miscellaneous group of negative alcohol and urine drug screens and the undocumented screen variables.

non-TTA patients. The RED and YELLOW coded patients were more likely associated not only with all substance use but also with more poly-drug use than GREEN coded patients. The median LOS for the TTA group was higher than for the non-TTA group, yet the TTA group had a higher probability of GREEN coded patients staying < 1 day, (OR 3.34 [95\% Cl 3.08, 3.62]), since they had more low-risk injuries (OR 4.07 [95\% $\mathrm{Cl} 3.78,4.39]$ ) (Figure 3).

\subsection{Substance Use}

In this study, the major substances used were alcohol, opiates, cannabinoids (THC), benzodiazepines (BZD), amphetamines, and cocaine sorted by the TTA coded groups and by the substance use cohorts. Positive urine drug screens reflected the following breakdown of illicit and prescription drug identification: $55.8 \%$ had one drug; $28.2 \%$ had two drugs; $16.1 \%$ had three to six drug combinations positive on admission.

Opiates $(1053,19.9 \%)$ and THC $(800,15.1 \%)$ were the major single drugs recorded, Figure 4. More THC and opiates were used by the $<20$ - and $20-$ 54.99 -year-old subset than by the $\geq 55$-year-old patients who were more likely to use BZD and opiates as single drugs. Major poly-drug consumption centered on opiates/THC (4.7\%) or opiates/BZD (4.6\%), Figure 5. In the UDS positive deceased population, the most frequent single drugs were BZD (21.4\%); THC (14.6\%); amphetamines (13.6\%) and opiates (12.6\%). The most frequent poly-drug combinations were BZD/amphetamines (4.9\%) and BZD/THC (2.9\%) with various other combinations. Poly-drug usage increased significantly in the three-four-five-six drug combinations between 2010-2013 and 2014-2018 (38\% to $62 \%), p<0.0001$.

\subsection{0-2013 versus 2014-2018}

For the screened TTA and non-TTA patients, there was a trend toward a significant decrease in alcohol + drugs and alcohol only use with more single drug/ 


\section{Single drug use by the trauma team activation codes 2010-2018}

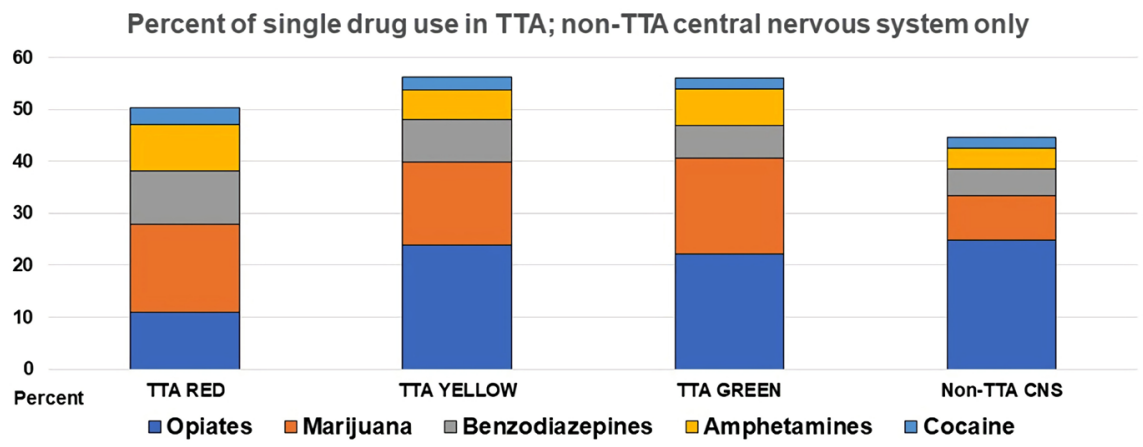

Figure 4. Single drug use by trauma team activation (TTA) color codes and non-TTA central nervous system (CNS) injury.

\section{Poly-drug use by the trauma team activation codes 2010-2018}

25

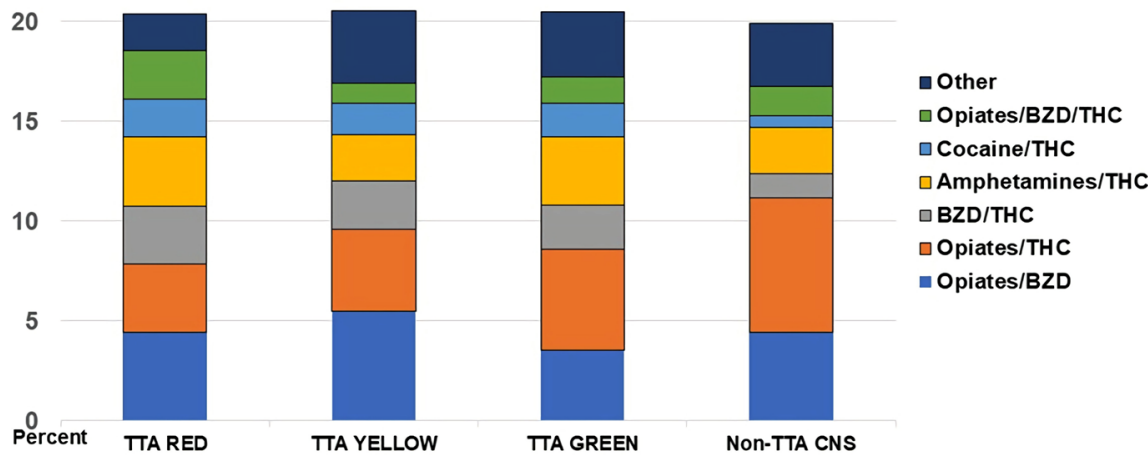

Figure 5. Poly-drug use by trauma team activation (TTA color codes and non-TTA central nervous system (CNS) injury.

poly-drug use in 2014-2018 than in 2010-2013. While alcohol use decreased from $58.0 \%$ to $42.0 \%$, single drug $(42.0 \%$ to $58.0 \%)$ and poly-drug use $(36.9 \%$ to $63.1 \%)$ increased significantly in 2014-2018, $p<0.0001$. The ISS did not increase between drug cohorts or time frames (2010-2013 to 2014-2018), $p=0.2$. However, there was a significant difference in ages and alcohol levels, $p<0.0001$; hospital charges, $p=0.017$; LOS, $p=0.02$ (Mann-Whitney U test). Hospital charges/patient increased for all drug cohorts between the two-time frames. As illicit drug use increased, Medicaid payments increased from $32.71 \%$ to $41.4 \%$, and self-pay decreased from $23.6 \%$ to $12.7 \%, p<0.001$. In the poly-drug cohort alone, Medicaid payments increased from $27.0 \%$ to $73.0 \%$ and self-pay decreased from $62.4 \%$ to $37.6 \%$ during these time periods.

\section{Discussion}

Our study has shown that the preponderance of substance users (52\%) was in 
the TTA group and $35.5 \%$ were in the non-TTA group. This finding substantiated the observations in the literature [4] [5]. The major single use drugs were opiates, benzodiazepines, THC and amphetamines. The major poly-drug combinations were opiates/THC, opiates/BZD, and THC/amphetamines. Both TTA and non-TTA groups had increased poly-drug use in 2014-2018 compared to 2010-2013. Patients in the miscellaneous group, who were TTA coded were more likely to be in the poly-drug cohort than in the other cohorts. Poly-drug usage has been reported as one of the most significant mortality risk factors in patients undergoing opioid substitution therapy [12] [13]. In 2013, Gudin et al. indicated that "the contribution of combination use of BZDs, alcohol, and/or other sedative agents to opioid-related morbidity and mortality is underappreciated, even when these agents are used appropriately..." to alleviate chronic pain [14]. Despite the potential significance of specific poly-drug combinations and alcohol use by trauma patients, most articles concentrate on opioid and single use illicit drugs; the literature does not combine alcohol and drug screens per patient but reports screening results separately [4] [15] [16]. The miscellaneous cohort of negative alcohol and drug screens and undocumented variable created a selection bias in data results. Vitale reviewed emergency room substance use studies and noted the lack of appropriate controls for the trauma patient: four had no controls; two had injured and non-injured; two compared violent crimes to accidental injuries; one tested to not tested patients; one emergency room patients to trauma patients who died; and one by age over and under 65-years [2]. Several recent studies reported only positive substance use tests results [3] [15] [16].

Compliance bias ensues when alcohol and drug screens are ordered but not performed on trauma patient arrivals in the medical facility due to: anuric or uncooperative patients, lack of a bladder catheter, uninformed staff, randomly missed orders, lost samples, busy house staff prioritizing sample collection, personnel assumptions, inadequate protocol training, or screen cost or legal concerns especially in drug testing [3] [4] [5] [8]. On the other end, trauma registrars may not be recording all the pertinent alcohol or urine screens, especially if the patients were transfers from another facility where those screens were performed but not recorded for the registrars to locate. Both selection bias by the frontline physicians and researchers, and compliance bias in obtaining alcohol blood levels and urine drug screens contribute to the issues with the trauma data analyzed.

In the United States, the National Trauma Data Bank (NTDB) was established between 2004-2006 by the American College of Surgeons Committee on Trauma (ACSCOT) as a national trauma registry for local and state accredited/designated trauma centers and is supported by the U.S. Health Resources and Services Administration (HRSA) [17]. As an accredited Level I trauma center, our institutional trauma registry case data is submitted to this national registry. Trauma registry substance use screens are reported in the following categories: positive, negative, not tested, unknown, unrecorded, non-applicable or blank. When 
trauma registry cases had not been tested or may have had positive or negative screens that were not recorded, a certain bias is created, and recognized in trauma and burn research [1]-[6]. Currently, there are no specifications for which trauma patients should be tested for alcohol and drug use. For this reason, the emphasis in this study was on the trauma team activation code, which is financially and medically required in trauma centers. Each trauma patient injury is visually assessed and triaged on arrival at the medical facility. This study has shown that most substance users were in the TTA group. Erdogan et al. reported that with an official trauma care policy, all TTA patients were to be screened for a blood alcohol concentration; alcohol screens increased from 33\% in 2000 to $85 \%$ in 2010 [18]. We summarized the relevant literature to show that trauma activation code status may be a solution to identify and increase alcohol and drug tests in trauma patients and decrease the inherent screening selection and compliance bias. Our study confirmed that TTA coded most of the substance users. While alcohol and drug screens are reported separately, there is no literature depicting the specific alcohol and drug combinations most frequently used among trauma patients, as described in this manuscript. One of the strengths of this study was the determination of the different prescription and illicit drug UDS combinations documented in trauma patients, indicating an increase in poly-drug use in combination with opioids. Increased screening would improve the opportunities for substance use prevention and treatment [10] [11].

\section{Limitations}

One of the limitations of this study was the lack of an unbiased "control" of negative alcohol and drug screens for comparison. The retrospective nature of the NTDB and study trauma registry contributed to the selection and compliance bias. Since this was a study performed in one urban trauma center, the results cannot be generalized.

\section{Conclusion}

This was a retrospective urban hospital trauma registry study about the correlation of the TTA code and substance use combinations in trauma patients between 2010 and 2018. The importance of this report is that while alcohol use decreased, the economic burden of poly-drug use mainly with opiates, THC, BZD, and amphetamines increased. Although not definitive because all trauma patients did not have a recorded alcohol and urine drug screens performed, this study showed a significant trend and pattern of increased poly-drug use. As noted in previous reports, TTA coded patients may be strong candidates for mandatory alcohol and drug screening upon trauma center arrival, while nonTTA patients may be more likely candidates for optional drug testing. The year 2020 will be a decisive marker for trends in substance use since the Covid-19 pandemic started. Continued marihuana state legalization may have stimulated more alcohol and poly-drug use, which will continue to impact patient care. Trauma surgeon development of more definitive protocols for required alcohol 
and UDS screens on trauma patients would improve databases for epidemiologic and research studies in the future.

\section{Conflicts of Interest}

The authors declare no conflicts of interest regarding the publication of this paper.

\section{References}

[1] London, J.A. and Battistella, F.D. (2007) Testing for Substance Use in Trauma Patients. Are We Doing Enough? The Archives of Surgery, 142, 633-638.

https://doi.org/10.1001/archsurg.142.7.633

[2] Vitale, S. and van de Mheen, D. (2006) Illicit Drug Use and Injuries: A Review of Emergency Room Studies. Drug and Alcohol Dependence, 82, 1-9. https://doi.org/10.1016/j.drugalcdep.2005.08.017

[3] Williams, F.N., Chrisco, L., Strassle, P.D., Laughon, S.L., Sljivic, S., Nurczyk, K., et al. (2020) Bias in Alcohol and Drug Screening in Adult Burn Patients. International Journal of Burns and Trauma, 10, 146-155.

[4] Dunham, C.M. and Chirichella, T.J. (2012) Trauma Activation Patients: Evidence for Routine Alcohol and Illicit Drug Screening. PLoS ONE, 7, e47999. https://doi.org/10.1371/journal.pone.0047999

[5] Langdorf, M.I., Rudkin, S.E., Dellota, K., Fox, J.C. and Munden, S. (2002) Decision Rule and Utility of Routine Urine Toxicology Screening of Trauma Patients. European Journal of Emergency Medicine, 9, 115-121. https://doi.org/10.1097/00063110-200206000-00003

[6] The NTDB Annual Report 2010. https://www.facs.org/ /media/files/quality\%20programs/trauma/ntdb/ntdbannualr eport2010.ashx

[7] The NTDB Annual Report 2016. https://www.facs.org/ /media/files/quality\%20programs/trauma/ntdb/ntdb\%20ann ual\%20report\%202016.ashx

[8] Warner, E.A., Walker, R.M. and Friedman, P.D. (2003) Should Informed Consent Be Required for Laboratory Testing for Drugs of Abuse in Medical Settings? The American Journal of Medicine, 115, 54-58. https://doi.org/10.1016/S0002-9343(03)00236-5

[9] Texas EMS Trauma and Acute Care Foundation (TETAF). http://tetaf.org/wp-content/uploads/2016/03/trauma-activation-guildelines.pdf

[10] Ornell, F., Moura, H.F., Scherer, J.N., Pechansky, F., Kessler, F.H.P. and von Diemen, L. (2020) The Covid-19 Pandemic and Its Impact on Substance Use: Implications for Prevention and Treatment. Psychiatry Research, 289, Article ID: 113096. https://doi.org/10.1016/j.psychres.2020.113096

[11] Boehnke, K.F., McAfee, J., Ackerman, J.M. and Kruger, D.J. (2020) Medication and Substance Use Increases among People Using Cannabis Medically during the Covid-19 Pandemic. International Journal of Drug Policy, Article ID: 103053. https://doi.org/10.1016/j.drugpo.2020.103053

[12] Gossop, M., Stewart, D., Treacy, S. and Marsden, J. (2002) A Prospective Study of Mortality among Drug Misusers during a 4-Year Period after Seeking Treatment. Addiction, 97, 39-47. https://doi.org/10.1046/j.1360-0443.2002.00079.x 
[13] Riley, E.D., Evans, J.L., Hahn, J.A., et al. (2016) A Longitudinal Study of Multiple Drug Use and Overdose among Young People Who Inject Drugs. American Journal of Public Health, 106, 915-917. https://doi.org/10.2105/AJPH.2016.303084

[14] Gudin, J.A., Mogali, S., Jones, J.D. and Comer, S.D. (2013) Risks, Management, and Monitoring of Combination Opioid, Benzodiazepines, and/or Alcohol Use. Postgraduate Medicine, 125, 115-130. https://doi.org/10.3810/pgm.2013.07.2684

[15] Gemma, V.A., Chapple, K.M., Goslar, P.W., Israr, S., Petersen, S.R. and Weinberg, J.A. (2018) Crisis under the Radar: Illicit Amphetamine Use Is Reaching Epidemic Proportions and Contributing to Resource Overutilization at a Level I Trauma Center. Journal of Trauma and Acute Care Surgery, 85, 953-959. https://doi.org/10.1097/TA.0000000000001984

[16] Cheng, V., Inaba, K., Johnson, M., Byerly, S., Jiang, Y., Matsushima, K., et al. (2016) The Impact of Pre-Injury Controlled Substance Use on Clinical Outcomes Following Trauma. Journal of Trauma and Acute Care Surgery, 81, 913-920. https://doi.org/10.1097/TA.0000000000001229

[17] National Trauma Data Bank (NTDB). https://www.facs.org/quality-programs/trauma/tqp/center-programs/ntdb/ntds/abo ut-ntds

[18] Erdovan, M., Kureshi, N., Karim, S.A., Tallon, J.M., Asbridge, M. and Green, R.S. (2018) Retrospective Analysis of Alcohol Testing in Trauma Team Activation Patients as a Canadian Tertiary Trauma Centre. BMJ Open, 8, e024190. https://doi.org/10.1136/bmjopen-2018-024190 